\title{
Brazilian Flavivirus Phylogeny Based on NS5
}

\section{Flávia Graciela Baleotti ${ }^{+}$, Marcos Lázaro Moreli , Luiz Tadeu Moraes Figueiredo}

Unidade Multidepartamental de Pesquisa em Virologia, Faculdade de Medicina de Ribeirão Preto, Universidade de São Paulo, Av. Bandeirantes 3900, 14049-000 Ribeirão Preto, SP, Brasil

In this work, a comprehensive phylogenetic study based on 600 base pair nucleotide and on putative 200 amino acid sequences of NS5 was carried out in order to establish genetic relationships among 15 strains of 10 Brazilian flaviviruses: Bussuquara, Cacipacore, dengue type 1, 2 and 4, Iguape, Ilheus, Rocio, Saint Louis encephalitis (SLE), and yellow fever. Phylogenetic trees were created by neighbor-joining and maximum parsimony methods. These trees showed Brazilian flaviviruses grouped into three main branches: yellow fever branch, dengue branch subdivided in types 1, 2 and 4 branches, and Japanese encephalitis virus (JEV) complex branch including SLE virus strains, Cacipacore, Iguape, Rocio, Ilheus and Bussuquara. Viruses transmitted by Aedes mosquitoes, such as dengue and urban yellow fever, that are also the only Flavivirus causing hemorrhagic fevers in Brazil, were grouped in the same cluster. Encephalitis associated viruses, transmitted by Culex mosquitoes such as JEV complex branch including SLE virus strains, Cacipacore, Iguape, Rocio, Ilheus and Bussuquara were also grouped in the same clade.

Key words: Brazilian Flavivirus - NS5 protein - Flavivirus phylogeny - Brazil

The family Flaviviridae comprises the genera Flavivirus, Pestivirus and Hepacivirus. Flaviviruses include a major importance group of arboviruses, which is responsible for considerable morbidity and mortality and may cause severe encephalitic disease, hemorrhagic fevers, hepatitis, and febrile illness in humans (Scaramozzino et al. 2001). Eleven flaviviruses are known to occur in Brazil: Bussuquara, Cacipacore, Dengue types 1, 2, 3 and 4, Iguape, Ilheus, Rocio, Saint Louis encephalitis (SLE) and yellow fever. Most of Brazilian flaviviruses are maintained in nature as sylvatic zoonoses that occasionaly can attack man and domestic animals in contact with ecosystems where such viruses occur (Figueiredo 2000). Brazilian urban populations have been struck by large dengue outbreaks due to population growth, urbanization, and spread of the main mosquito vector, Aedes aegypti. Dengue disease became a major emerging disease with the cocirculation of three serotypes, increased frequency of epidemics (536,398 dengue cases reported in 1998), and the increasing occurrence of hemorrhagic dengue cases (575 DHF/DSS cases were reported in 2001 with a total of 28 letality cases). Yellow fever is the prototype member of the genus Flavivirus, it is transmitted by Haemagogus mosquitoes in South America. It causes a viral hemorragic fever with a higher than $50 \%$ case fatality rate (Mutebi et al. 2001). Dozens of sylvatic cases, many of them fatal, are reported annually in the Amazon, central plateau and Pantanal regions of Brazil (Batista et al. 2001). Furthermore, there is a permanent risk of yellow fever urbaniza-

This work was supported by State of São Paulo Research Agency (Fapesp), Brazil.

${ }^{+}$Corresponding author. Fax: +55-16-633.6695. E-mail: flaviagraci@zipmail.com.br.

Received 3 May 2002

Accepted 6 March 2003 tion in Brazilian cities caused by the constant arrival of viremic patients infected in sylvatic transmission areas (Batista et al. 2001).

Other sylvatic Brazilian flaviviruses are Bussuquara, Cacipacore, Ilheus, Iguape, Rocio and SLE. Bussuquara and Ilheus viruses are the causatives of acute febrile illness. Rocio virus caused an outbreak with more than 1000 reported cases of encephalitis, between 1973 and 1980, with 100 deaths and more than 200 sequelae cases, in the Ribeira Valley Region, Southeastern Brazil. One case of fever and jaundice caused by SLE virus was described. Encephalitis caused by SLE virus was not reported in Brazil. Cacipacore virus was isolated from a bird in the Amazon region, and Iguape virus was isolated from a sentinel mouse in Southern Brazil; however, they have not been described as causative of human disease (Figueiredo 2000).

Flavivirus enveloped particles have 40 to $60 \mathrm{~nm}$ diameter, and include the nucleocapsid containing approximately 11,000 nucleotides single-stranded, positive-sense RNA. The genome is composed of a short 5' noncoding region, a single open reading frame (ORF) containing more than 10,000 nucleotides, and a 3' noncoding terminus. The long ORF encodes 3 structural proteins, of the viral capsid (C), pre-membrane (preM), and envelope (E), and 7 nonstructural proteins NS1, NS2A, NS2B, NS3, NS4A, NS4B, NS5.

The last protein encoded in the ORF is NS5, the largest and most highly conserved Flavivirus protein. NS5 is a basic protein, lacking any long hydrophobic stretches, and it is assumed to be the Flavivirus RNA-dependent RNA polymerase (RdRp). NS5 is a key enzyme for the viral RNA replication. Flavivirus NS5 amino acid comparison with RNA polymerases of other viruses revealed six common conserved sequence motifs including four characteristic polymerase motifs (A, B, C and D). The C motif (GDD) is the signature for RdRp and is highly conserved in NS5. The N-terminal domain of NS5 is homolo- 
gous to methyltransferase regions implicated in Sadenosylmethionine binding. It has been suggested that this domain may be involved in methylation of the 5' cap structure. Therefore, NS5 is at least bifunctional, possessing both methyltransferase and RNA polymerase activities (Koonin 1993).

In the last 20 years, the molecular revolution in biology has given birth to molecular taxonomy. This new taxonomy provides important information about evolutionary relationships based on nucleotide sequences of virus genes. Here, we report a molecular epidemiology study based on a 600 nucleotide sequence of the NS5 gene of 15 Brazilian flaviviruses.

\section{MATERIALS AND METHODS}

Viruses - The 15 Brazilian flaviviruses analyzed in the study were previously reported (Batista et al. 2001). Dengue, Ilheus, Rocio, and SLE viruses were originally isolated from patients (Lopes et al. 1978, Osanai et al. 1983, Schatzmayr et al. 1976, Figueiredo et al. 1994, Vasconcelos et al. 1993, 1995). The other viruses were isolated from wild animals (Lopes et al. 1979, Pinheiros et al. 1981).

All viral strains were obtained from mouse brain tissue suspensions or from infected Aedes albopictus C6/36 cell fluids. Viruses were passaged once by intracerebral inoculation in baby mice, the mouse infected brains were collected, diluted 1:20 in Leibowitz L15 medium and inoculated into C6/36 A. albopictus cell monolayers. The infected cell cultures were held for $6-7$ days, at $28^{\circ} \mathrm{C}$, and were stored at $-70^{\circ} \mathrm{C}$ after infection confirmation by indirect immunofluorescence test using Flavivirus mouse immune ascitic fluids (Figueiredo 1990).

Viral RNA extraction - RNA was extracted from infected tissue culture fluids using the QIAmp Viral RNA Extraction Kit (Qiagen, USA), following the manufacturer's instructions.

Reverse transcription-PCR - The FG1 and FU1RC primers were used in the RT-PCR. These primers amplify about 720 nucleotides of the NS5 gene of the flaviviruses and were previously reported by Kuno et al. (1998) (FU1RC) and Fulop et al. (1993) (FG1).

RT reactions were performed in $12 \mu \mathrm{l}(\sim 1.5 \mu \mathrm{g})$ of the extracted RNA suspensions, including $0.5 \mu \mathrm{l}(100 \mathrm{U})$ of reverse transcriptase (Pharmacia USA), $1 \mu \mathrm{l}$ (36 U) of RNAse inhibitor (Pharmacia USA), $1 \mu \mathrm{l}(100 \mathrm{mM})$ of the FU1RC reverse primer, $1 \mu 1(0.1 \mathrm{mM})$ of each one of the 4 deoxynucleoside triphosphates, and $4 \mu \mathrm{l}$ of $5 \mathrm{X}$ buffer (250 mM Tris pH 8.3, $15 \mathrm{mM} \mathrm{MgCl}_{2}, 375 \mathrm{mM} \mathrm{KCL}, 50 \mathrm{mM}$ DTT). The mixture was incubated at $37^{\circ} \mathrm{C}$ for $1 \mathrm{~h}$. PCR reactions were performed in $50 \mathrm{ml}$ mixtures containing $3.5 \mu 1$ of the RT mixture, $1.25 \mu 1$ (1U) of Taq DNA polymerase (Biotools Spain), $1 \mu \mathrm{l}(0.1 \mathrm{mM}$ ) of each one of the 4 deoxynucleoside triphosphates, $1 \mu \mathrm{l}(100 \mathrm{mM})$ of FG1 sense primer, $1 \mu \mathrm{l}(100 \mathrm{mM})$ of FU1RC reverse primer, $5 \mu \mathrm{l}$ of $10 \mathrm{X}$ buffer [(Tris- $\mathrm{HCl} 75 \mathrm{mM} \mathrm{pH} 9.0, \mathrm{MgCl}_{2} 2 \mathrm{mM}$, $\left.\left(\mathrm{NH}_{4}\right)_{2} \mathrm{SO}_{4} 20 \mathrm{mM}\right)$ ], and $37.5 \mu \mathrm{l}$ of DEPC water. The PCR conditions were: $94^{\circ} \mathrm{C}$ for $1 \mathrm{~min}, 45^{\circ} \mathrm{C}$ for $3 \mathrm{~min}$, and $72^{\circ} \mathrm{C}$ por $1 \mathrm{~min}$. The amplification products were identified under UV light transilumination, based on their molecular weights, after electrophoresis in $2 \%$ agarose gel, and with ethidium bromide staining.
Nucleotide sequencing - Products of PCR amplification were purified using the PCR Preps DNA Purification Resin (Promega, USA), and suspended in $50 \mu 1$ of sterile distilled water. The purified DNA amplicons were treated with exonuclease I ( $5 \mu 1$ of PCR product and $5 \mu$ l of Exo I) by incubation at $80^{\circ} \mathrm{C}$ for $15 \mathrm{~min}$, following $37^{\circ} \mathrm{C}$ for 15 $\mathrm{min}$, and finally were stored at $-20^{\circ} \mathrm{C}$.

The purified DNAs were sequenced using the Thermo Sequenase Kit (Amersham). Briefly, Cy5.5 dye-labelled ddNTP terminators were used in the sequencing PCR and unincorporated dye terminators were removed by ethanol precipitation with $0.25 \mathrm{vol}$ of $7.5 \mathrm{M}$ ammonium acetate and 3 vol of $100 \%$ ethanol. Following, the precipitates were suspended in $6 \mathrm{ml}$ of formamide loading dye and $2 \mu 1$ of each sample was loaded into separate lanes of a denaturing $6 \%$ polyacrylamide gel containing urea and processed in a Personal seq 4X4 (Pharmacia,USA) nucleotide sequencing machine.

Phylogenetic analysis - Virus sequences of 600 nucleotides and of the 200 putative amino acids of NS5 gene of the 15 Brazilian Flavivirus, also including reported sequences from dengue type 1 Singapore strain virus (DEN1-S, Genbank NC87512), dengue type 2 Jamaica strain virus (DEN2-J, Genbank NC20558), dengue type 4 Guine Bissau virus (DEN4-GB Genbank NC 002640) and Japanese encephalitis strain virus (JEV, Genbank NC001437), were aligned by the Higgins and Sharp algorithm Clustal W (D Higgins, J Thompson and T Gibson). The multiple sequence alignment program Clustal W was used to obtain optimal nucleotide or amino acid sequence alignment files. Phylogenetic trees were performed by neighbor-joining method using Clustal W (D Higgins, J Thompson and T Gibson), and by parsimony analysis using the PAUP* program version 4.0b3 (Sinauer,USA), employing bootstrap method with heuristic search option with TBR branch-swapping algorithm, maximum parsimony as optimality criterion, and gaps treated as missing entries. Nucleotide parsimony trees have ancestor standard included in analysis and the tree is rooted. Amino acid parsimony tree is unrooted. Clade support for the maximum parsimony tree was assessed by bootstrap re-sampling analysis (100 replicatives), and considered only bootstrap 50\% majority-rule consensus trees.

\section{RESULTS}

Six hundred nucleotides of NS5 of each one of the 15 strains of Brazilian flaviviruses, also including the nucleotides of 4 other flaviviruses were aligned.

Neighbor-joining phylogenetic trees based on the multiple alignment of 600 base pair nucleotides and on the 200 putative amino acids of NS5 of 15 Brazilian flaviviruses, also including sequences of 4 other flaviviruses are shown in Fig. 1A, B.

Parsimony phylogenetic trees based on multiple alignment of the 600 nucleotide sequences and the putative 200 amino acid sequences of the NS5 of 15 Brazilian flaviviruses, also including sequences of 4 other flaviviruses, are shown in Fig. 2A, B respectively. 


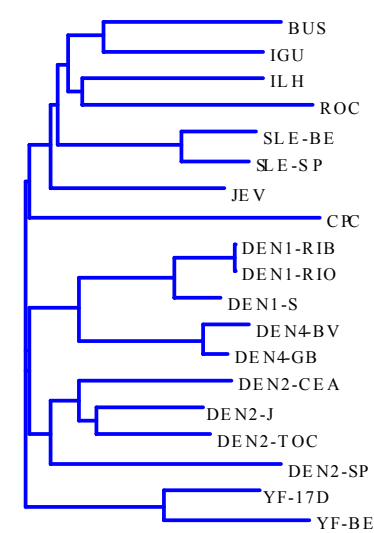

A

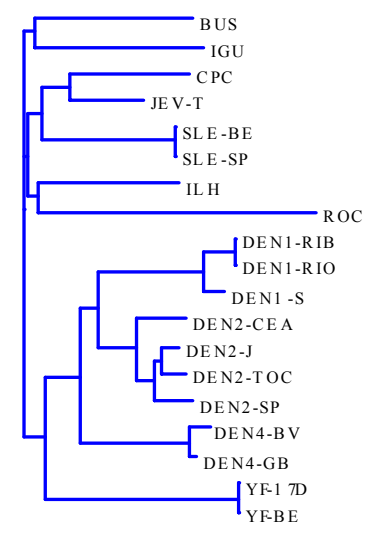

B

Fig. 1: neighbor-joining phylogenetic trees of a sequence of 600 nucleotides (A) and 200 amino acids (B) of the NS5 gene, of 15 Brazilian Flavivirus, also including DEN1-S, DEN2-J, DEN4-GB and JEV.

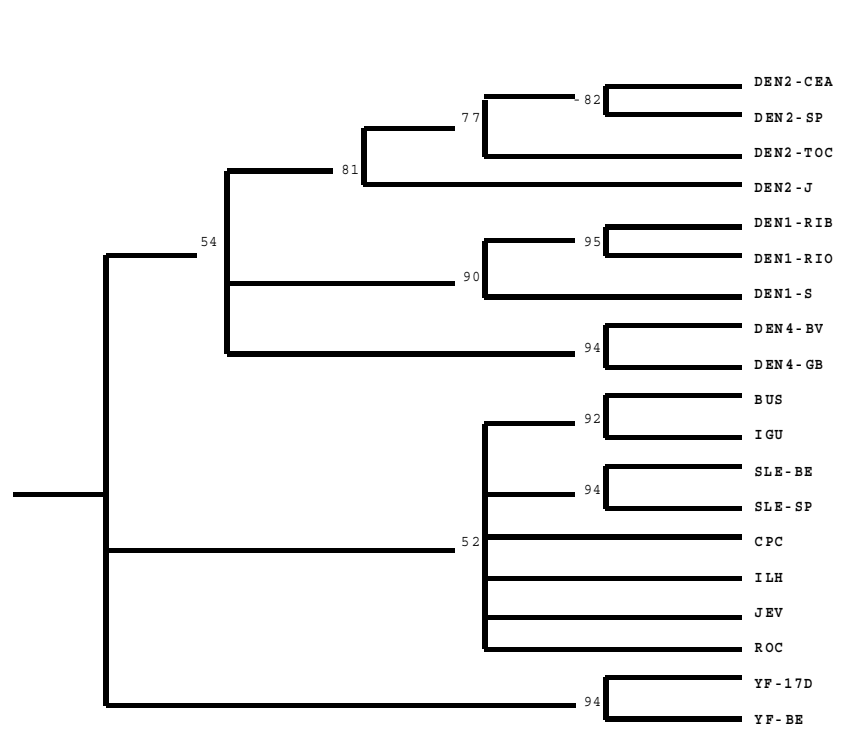

A

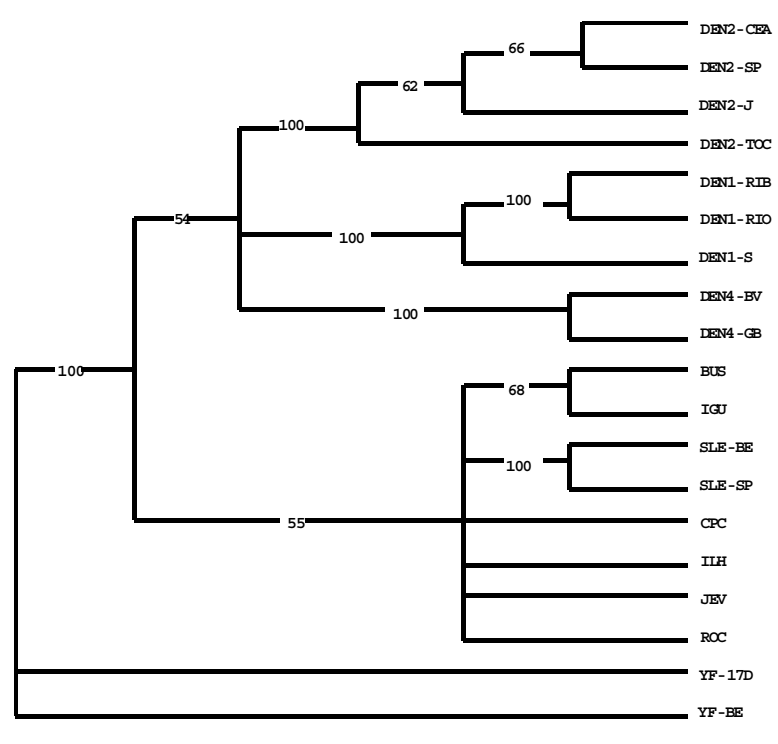

B

Fig. 2: phylogenetic trees, obtained by parsimony method, of a sequence of 600 nucleotides (A) and of 200 amino acids (B) of the NS5 gene, of 15 Brazilian Flavivirus, also including DEN1-S, DEN2-J, DEN4-GB and JEV viruses. Numbers below clades refer to bootstrap proportions (BP); BPs $<50$ are not indicated.

\section{DISCUSSION}

The primer pair including FG1 and the FU1RC showed a suitable broad performance among Brazilian flaviviruses generating about $723 \mathrm{bp}$ amplicons from NS5 of all the 15 studied viruses.

In order to obtain reproducible phylogenetic trees and reliable branching, two methods were used for virus nucleotide and amino acid alignment of sequences and dendogram generation (multiple alignment/ neighbor-joining and pairwise/parsimony). Four virus sequences from GenBank (USA) were included in the phylogenetic analysis to provide information on divergence levels in comparison to other flaviviruses.
All the neighbor-joining and parsimony trees, based on aligned nucleotide and amino acid sequences of NS5 gene, showed the Brazilian Flavivirus grouped into three main branches: dengue branch subdivided in types 1,2 and 4 branches, JEV complex branch including SLE, Cacipacore, Bussuquara, Rocio, Ilheus, Iguape, and yellow fever branch.

The branching patterns leading to clades were found to be closely similar between the neighbor-joining and parsimony trees based on nucleotide as well as amino acid sequences.

Cacipacore virus was considered ungrouped based on classical phylogenetic studies done by cross-neutralization tests. In our study, in both neighbor-joining and 
parsimony trees, Cacipacore virus appears as part of the JEV complex. Our data corroborates those reported by Batista et al. (2001) studying the 124 NS5 final nucleotides of the NS5 gene and the following 129 nucleotides of the 3 ' noncoding region of Brazilian flaviviruses. The authors found Cacipacore virus as part of the JEV complex in a maximum parsimony phylogenetic tree. A similar inclusion of Cacipacore in the JEV clade was also observed by Kuno et al. (1998) studying a $1 \mathrm{~Kb}$ segment at the 3' terminus of the NS5 gene.

Rocio virus was also considered ungrouped based on classical phylogenetic studies done by cross-neutralization tests. In our neighbor-joining and parsimony phylogenetic trees, Rocio virus appears in a branch of the JEV clade. Our results agree with those of Batista et al. (2001) and Kuno et al. (1998) who also observed Rocio virus in the JEV clade in their neighbor-joining phylogenetic trees.

Bussuquara and Iguape viruses appear in a same branch of the JEV clade in our neighbor-joining and parsimony phylogenetic trees. In the study of Batista et al. (2001), Iguape virus appears as an ungrouped branch in the phylogenetic tree. This difference on grouping Iguape virus in the JEV complex is probably related to the different genome regions that were analyzed in both studies. In the study of Kuno et al. (1998), Bussuquara and Iguape viruses were both included in the JEV complex.

In our study, Ilheus virus was grouped with Rocio virus in the trees corroborating the findings reported by Kuno et al. (1998) in the same kind of tree.

All our phylogenetic trees included yellow fever viruses and dengue viruses in two separated clades. These yellow fever and dengue clades have been consistently shown in most of the Flavivirus phylogenetic studies, analyzing different genome regions. Dengue and yellow fever are traditionally recognized as major serocomplexes (clades) in the genus Flavivirus which was corroborated by our phylogenetic trees showing both dengue and yellow fever topologies, with high bootstraps.

Phylogenetic analysis of Brazilian Flavivirus could be important for a better understanding of hosts and natural cycles of these viruses as well as for a better understanding of clinical diseases and pathogenesis based on correlation among viruses belonging to a same clade or cluster. Viruses transmitted by Aedes mosquitoes, such as dengue and urban yellow fever, that are also the only Flavivirus causing hemorrhagic fevers in Brazil, were grouped in the same cluster. Viruses transmitted by Culex mosquitoes and causing encephalitis as Rocio and SLE virus were grouped in the JEV complex. Viruses included in the same branch of JEV complex, such as Iguape and Cacipacore, despite of being never described as causing human diseases, and Bussuquara and Ilheus viruses, despite of being described as causing encephalitis in human, based on their close phylogenetic relationships with Rocio and SLE virus make, we infer they could be potential causatives of human infections of the central nervous system.

Nevertheless, we believe that our molecular classification produces the smallest amount of discrepancy com- pared with serologic classification and together, the two methods would greatly improve our understanding of the relationship among the Brazilian Flavivirus and a better understanding of clinical diseases and pathogenesis of Brazilian Flavivirus based on correlation among viruses belonging to a same clade or cluster.

\section{REFERENCES}

Batista WC, Kashima S, Marques AC, Figueiredo LTM 2001. Phylogenetic analysis of Brazilian Flavivirus using nucleotide sequences of parts of NS5 gene and 3'non-coding regions. 2001. Virus Res 75: 35-42.

Figueiredo LTM 1990. Uso de células de Aedes albopictus C6/ 36 na propagação e classificação de arbovírus das famílias Togaviridae, Flaviviridae, Bunyaviridae e Rhabdoviridae. Rev Soc Bras Med Trop São Paulo 23: 13-18.

Figueiredo LTM 1994. Os arbovírus do Brasil. Anais da Segunda Reunião Anual da Sociedade Brasileira de Pesquisadores Nikkeis, São Paulo, p. 45-58.

Figueiredo LTM 2000. The Brazilian flaviviruses. Microbes and Infections 2: 1643-1649.

Fulop L, Barret ADT, Phillpotts R, Martin K, Leslie D, Titbal R 1993. Rapid identification of flaviviruses based on conserved Ns5 gene sequences. J Virol Methods 44:179-188.

Koonin EV 1993. Computer-assisted identification of a putative methyltransferase domain in NS5 protein of flaviviruses and lambda 2 protein of reovirus. J Gen Virol 74: 733-740.

Kuno G, Chang GJJ, Tsuchiya KR, Karabatsos N, Cropp B 1998. Phylogeny of the genus Flavivirus. J Virol 72: 73-83.

Lopes OS, Coimbra TLM, Sacchetta LA, Calisher CH 1978. Emergence of a new arbovirus disease in Brazil. 1. Isolation and characterization of the etiologic agent, Rocio virus. $\mathrm{Am}$ J Epidemiol 107: 444-449.

Lopes OS, Sacchetta LA, Coimbra TLM, Pereira LE 1979. Isolation of St. Louis encephalitis virus in south Brazil. Am J Trop Med Hyg 28: 583-585.

Mutebi JP, Wang H, Li Li, Bryant JE, Barret ADT 2001. Phylogenetic and evolutionary relationships among yellow fever virus isolates in Africa. J Virol 75: 6999-7008.

Osanai CH, Travassos da Rosa, Tang AT 1983. Surto de dengue em Boa Vista, Roraima: Nota Prévia. Rev Inst Med Trop São Paulo 23: 53-54.

Pinheiro FP, LeDuc JW, Travassos da Rosa AP, Leite CF 1981. Isolation of St. Louis encephalitis virus from a patient in Belém, Brazil. Am J Trop Med Hyg 30: 145-148.

Scaramozzino N, Crance JM, Jouan A, De briel DA, Stoll F, Garin D 2001. Comparison of Flavivirus universal primer pairs and development of a rapid, highly sensitive heminested reverse transcription-PCR assay for detection of flaviviruses targeted to a conserved region of the NS5 gene sequences. J Clin Microbiol 39: 1922-1927.

Schatzmayr PHA, Nogueira RMR, Travassos da Rosa AP 1976. An outbreak of dengue virus at Rio de Janeiro. Mem Inst Oswaldo Cruz 8: 245-246.

Vasconcelos PFC, Travassos da Rosa ES, Freitas RB, Dégallier N, Rodrigues SG, Travassos da Rosa APA 1993. Epidemia de febre clássica de dengue causada pelo sorotipo $2 \mathrm{em}$ Araguaina, Tocantins, Brasil. Rev Inst Med Trop São Paulo 35: 141-148.

Vasconcelos PFC, Travassos da Rosa JFS, Guerreiro SC, Dégallier N, Travassos da Rosa ES, Travassos da Rosa APA 1999. Primeiro registro de epidemias causadas pelo vírus Oropouche nos estados do Maranhão e Goiás, Brasil. Rev Inst Med Trop São Paulo 31: 271-278. 